\title{
AN ELEMENTARY TRANSFORMATION OF A SPECIAL UNIMODULAR VECTOR TO ITS TOP COEFFICIENT VECTOR
}

\author{
RAVI A. RAO
}

\begin{abstract}
Let $R$ be a commutative ring, $\mathbf{v}(X)$ a unimodular $n$-vector $(n \geqslant 3)$ over $R[X]$. Suppose the leading coefficients in $\mathrm{v}(X)$ form a unimodular vector $L(\mathrm{v})$ over $R$. Then some element in $E_{n}(R[X])$ will transform $\mathrm{v}(X)$ to $L(\mathrm{v})$.
\end{abstract}

1. Introduction and motivation. For a commutative ring $R, E_{n}(R)$ will denote the subgroup of the determinant one matrices $\operatorname{Sl}_{n}(R)$ generated by $E_{i j}(\lambda)=I_{n}+\lambda e_{i j}$, where $I_{n}$ denotes the $n \times n$ identity matrix, $1 \leqslant i \neq j \leqslant n, \lambda \in R$, and $e_{i j}$ is the matrix whose $(i, j)$ th entry is 1 and all other entries are zeros. If $\alpha \in E_{n}(R)$, call it an elementary matrix.

A vector $\mathbf{v}=\left(v_{1}, \ldots, v_{n}\right) \in R^{n}$ is called unimodular if there is a vector $\mathbf{w}=$ $\left(w_{1}, \ldots, w_{n}\right) \in R^{n}$ with $\mathbf{v} \cdot \mathbf{w}^{t}=\sum_{i} v_{i} w_{i}=1 . \mathrm{Um}_{n}(R)$ will denote the set of all unimodular vectors in $R^{n}$. Clearly, $E_{n}(R)$ acts on $\operatorname{Um}_{n}(R)$. Denote by " $\equiv$ $\left(\bmod E_{n}(R)\right)$ ' vectors in the same orbit under this action.

For a polynomial $f \in R[X], L(f)$ will denote the leading coefficient of $f$.

A vector $\mathbf{v}=\left(v_{1}, \ldots, v_{n}\right) \in \mathrm{Um}_{n}(R[X])$ is called a special unimodular vector if $L(\mathbf{v})=\left(L\left(v_{1}\right), \ldots, L\left(v_{n}\right)\right) \in \mathrm{Um}_{n}(R)$.

The principal aim of this short note is to point out

ThEOREM. Let $\mathrm{v} \in \mathrm{Um}_{n}(R[X]), n \geqslant 3$, be a special unimodular vector. Then $\mathbf{v} \equiv L(\mathbf{v})\left(\bmod E_{n}(R[X])\right)$.

This simplifies and extends a result of A. A. Suslin in [S, Proposition 2.4].

The theorem settles affirmatively a question raised by M. P. Murthy during discussions, viz. if $\mathrm{v} \in \operatorname{Um}_{n}(R[X]), n \geqslant 3$, has a monic polynomial as one of its entries, then is $\mathbf{v} \equiv \mathbf{e}_{1}=(1,0, \ldots, 0)\left(\bmod E_{n}(R[X])\right)$ ?

Murthy's question was raised in relation to the following problem on the efficient generation of ideals in polynomial rings.

Problem. Let $R$ be a noetherian ring, and $I$ an ideal in the polynomial ring $R[X]$ containing a monic polynomial. Let I satisfy the following condition on its conormal bundle; viz the $R / I$-module $I / I^{2}$ :

$$
\begin{aligned}
\mu_{R / I}\left(I / I^{2}\right) & \stackrel{\text { def }^{n}}{=} \text { the least number of generators of } I / I^{2} \text { as a } R / I \text {-module } \\
& \geqslant \operatorname{dim}(R[X] / I)+2 .
\end{aligned}
$$

Then is $\mu(I)=\mu\left(I / I^{2}\right)$ ?

Received by the editors September 16, 1983 and, in revised form, March 23, 1984.

1980 Mathematics Subject Classification. Primary 13D15; Secondary 13B25, 20G35.

(C)1985 American Mathematical Society $0002-9939 / 85 \$ 1.00+\$ .25$ per page 
Recently, S. Mandal [M] settled the above problem affirmatively. Earlier, N. Mohan Kumar's reductions in [MK] had established a surjection $P \rightarrow I \rightarrow 0$ with $P$ a projective $R[X]$-module of rank $\mu\left(I / I^{2}\right)$. By utilizing the finer information available now by our theorem, we can considerably simplify the treatment in [MK] and show that $I$ is the onto image of a free module of rank $\mu\left(I / I^{2}\right)$, thereby providing a quick alternative solution to the above problem.

Let me mention two applications of this problem.

COROllaRY 1. Let $R$ be a noetherian ring, and $I$ an ideal in $R\left[X_{1}, \ldots, X_{n}\right]$, with height $I>\operatorname{dim} R$, and $\mu\left(I / I^{2}\right) \geqslant \operatorname{dim}\left(R\left[X_{1}, \ldots, X_{n}\right] / I\right)+2$. Then $\mu(I)=\mu\left(I / I^{2}\right)$.

COROLlaRY 2. Let $X$ be an irreducible nonsingular affine variety of dimension $d$ over a field $k$. Let $Y$ be a closed subset in $X \times \mathbf{A}_{k}^{n}$ which has pure dimension one. Assume $n \geqslant 2$. Then if the conormal sheaf of $Y$ in $X \times \mathbf{A}_{k}^{n}$ is trivial, $Y$ is a complete intersection in $X \times \mathbf{A}_{k}^{n}$.

Corollary 1 is immediate due to a lemma of Bass [B, Lemma 3]. Corollary 2 was established in [BR, Corollary 3.3].

2. The main theorem. $\mathrm{v} \equiv L(\mathrm{v})\left(\bmod E_{n}(R[X])\right)$.

The case when $R$ is a local ring was dealt with by Suslin-see [ $\mathbf{L}$, Chapter III, Lemma 2.8] for instance. For the sake of completeness we include a proof.

(2.1) Proposition (Suslin). Let $R$ be a local ring and $\mathrm{v} \in \mathrm{Um}_{n}(R[X]), n \geqslant 3$, have an entry which is an associate of a monic polynomial. Then $\mathbf{v} \equiv \mathbf{e}_{1}$ $\left(\bmod E_{n}(R[X])\right)$.

Proof. Without any loss of generality, let $\mathbf{v}=\left(v_{1}, \ldots, v_{n}\right) \in \operatorname{Um}_{n}(R[X])$, with $v_{1}$ an associate of a monic polynomial. Then $A=R[X] /\left(v_{1}(X)\right)$ is semilocal, and so $\mathrm{Sl}_{n-1}(A)=E_{n-1}(A)$. Hence, going modulo $\left(v_{1}\right)$ and then lifting, we may transform $\mathbf{v}$ to $\left(v_{1}, 1+v_{1} v_{2}^{\prime}, v_{1} v_{3}^{\prime}, \ldots, v_{1} v_{n}^{\prime}\right)$ by an elementary action, where $v_{i}^{\prime} \in R[X]$ for $2 \leqslant i \leqslant n$. It is now easy to complete the proof.

To reduce to the local case, we are led by ideas of L. N. Vaserstein-see [L, Theorem 2.4]-to consider the "local-global" nature of the action of $E_{n}(R[X])$, $n \geqslant 3$, in sending a vector $\mathbf{v} \in \operatorname{Um}_{n}(R[X])$ to its projection $\mathbf{v}(0)$. For this we shall use the following slight variant of a lemma of Suslin [S, Lemma 3.4], which can be proved in an identical fashion.

(2.2) Lemma (Suslin). Suppose $\alpha \in E_{n}\left(R_{s}[X]\right), \alpha(0)=I_{n}$, for some $s \in R$. Then there exists a natural number $k$ such that for any $r_{1}, r_{2} \in R[X]$ with $r_{1}-r_{2} \in s^{k} R[X]$, $\alpha\left(r_{1} X\right)^{-1} \alpha\left(r_{2} X\right) \in E_{n}(R[X])$.

(2.3) Local - Global Theorem. Let $\mathbf{v} \in \mathrm{Um}_{n}(R[X]), n \geqslant 3$. Suppose that for all $\mathrm{m} \in \operatorname{Max} R, \mathbf{v} \equiv \mathbf{v}(0)\left(\bmod E_{n}\left(R_{\mathrm{m}}[X]\right)\right)$. Then $\mathbf{v} \equiv \mathbf{v}(0)\left(\bmod E_{n}(R[X])\right)$.

Proof. Let $J=\left\{s \in R \mid \mathbf{v} \equiv \mathbf{v}(0)\left(\bmod E_{n}\left(R_{s}[X]\right)\right)\right\}$ denote the 'Quillen ideal' of v. In view of the assumption, it suffices to show that this set is actually an ideal. 
Clearly, one only needs to check that $s_{1}, s_{2} \in J \Rightarrow s_{1}+s_{2} \in J$. Inverting $s_{1}+s_{2}$, we may assume $s_{1}+s_{2}=1$.

Let $\sigma_{i} \in E_{n}\left(R_{s_{i}}[X]\right), i=1,2$, with $\sigma_{i} \mathbf{v}=\mathbf{v}(0)$. We may assume $\sigma_{i}(0)=I_{n}, i=1,2$. Let $\alpha=\sigma_{2} \sigma_{1}^{-1} \in E_{n}\left(R_{s_{1} s_{2}}[X]\right)$. By (2.2) there exists $k>0$ such that $\alpha(a X) \in$ $E_{n}\left(R_{s_{2}}[X]\right)$ if $s_{1}^{k} \mid a$, and $\alpha(a X)^{-1} \alpha(X) \in E_{n}\left(R_{s_{1}}[X]\right)$ if $s_{2}^{k} \mid(1-a)$. Since $\left(s_{1}, s_{2}\right)=1$, given any integer $k$, such an $a$ is readily available.

Observe that, since $\mathbf{v}(0)$ is a constant vector and $X \rightarrow a X$ is $R$-linear, $\alpha(X) \alpha(a X)^{-1}$ preseves $\mathbf{v}(0)$ ! But then

$$
\sigma_{2} \sigma_{1}^{-1}=\alpha(X)=\alpha(a X)\left(\alpha(a X)^{-1} \alpha(X)\right)=\beta_{2} \beta_{1}
$$

with $\beta_{i} \in E_{n}\left(R_{s_{i}}[X]\right), i=1,2$, and $\beta_{i} \mathbf{v}(0)=\mathbf{v}(0)$. Replacing $\sigma_{2}$ by $\beta_{2}^{-1} \sigma_{2}, \sigma_{1}$ by $\beta_{1} \sigma_{1}$, we obtain a $\gamma \in \mathrm{Sl}_{n}(R[X])$ with $\gamma \mathbf{v}=\mathbf{v}(0)$, and $\gamma_{s_{i}}=\sigma_{i}, i=1,2$. Since $\gamma$ is "locally" elementary, by [S, Theorem 3.1], $\gamma \in E_{n}(R[X])$. Thus, $J$ is an ideal.

A Horrock's-like argument now completes the proof of the main theorem.

(2.4) ThEOREM. Let $\mathbf{v} \in \mathrm{Um}_{n}(R[X]), n \geqslant 3$, be a special unimodular vector. Then $\mathbf{v} \equiv L(\mathbf{v})\left(\bmod E_{n}(R[X])\right)$.

Proof. Regard $\mathbf{v}=\left(v_{1}, \ldots, v_{n}\right) \in \mathrm{Um}_{n}(R[X-1]) ! \quad$ By $(2.1), \quad \mathbf{v}_{\mathrm{m}} \equiv \mathbf{v}(1)$ $\left(\bmod E_{n}\left(R_{\mathrm{m}}[X-1]\right)\right)$ for all $\mathrm{m} \in \operatorname{Max}(R)$ since $\mathrm{v}$ is special. Therefore, by the Local-Global Theorem $(2.3), \mathrm{v} \equiv \mathrm{v}(1)\left(\bmod E_{n}(R[X-1])\right)$. It thus suffices to show that $\mathbf{v}(1) \equiv L(\mathbf{v})\left(\bmod E_{n}(R)\right)$.

Let $m_{i}=\operatorname{deg} v_{i}$, and put $w_{i}=X^{-m_{i}} v_{i} \in R\left[X^{-1}\right]$, for $1 \leqslant i \leqslant n$. Then $\mathbf{w}=$ $\left(w_{1}, \ldots, w_{n}\right) \in \mathrm{Um}_{n}\left(R\left[X^{-1}\right]\right)$, as $\mathbf{w}$ is unimodular after inverting $X^{-1}$, and $\mathbf{w}(0)=$ $L(v) \in \operatorname{Um}_{n}(R)$. Applying (2.1) and (2.3) as above we get

$$
\mathbf{w} \equiv \mathbf{w}(0)=L(\mathbf{v}) \quad\left(\bmod E_{n}\left(R\left[X^{-1}\right]\right)\right) .
$$

Putting $X^{-1}=1$, we get

$$
\mathbf{w}(1)=\mathbf{v}(1) \equiv L(\mathbf{v}) \quad\left(\bmod E_{n}(R)\right) !
$$

(2.5) COROLlaRY. Let $\mathrm{v} \in \mathrm{Um}_{n}(R[X]), n \geqslant 3$, with some entry of $\mathrm{v}$ an associate of a monic polynomial. Then $\mathbf{v}$ can be completed to an elementary matrix $\alpha \in E_{n}(R[X])$.

(2.6) REMARK. We indicate another interesting proof of (2.4) which arose out of trying to understand why the proof of the theorem in $[\mathbf{M}]$ works.

Think of $\mathrm{v} \in \mathrm{Um}_{n}\left(R\left[X, T, T^{-1}\right]\right)$ !. Consider the $R$-linear automorphism $\varphi$ of $R\left[X, T, T^{-1}\right]$ which fixes $T$ and sends $X$ to $X-T+T^{-1}$. Clearly, it suffices to show $\varphi(\mathbf{v}) \equiv L(\mathbf{v})\left(\bmod E_{n}\left(R\left[X, T, T^{-1}\right]\right)\right)$. The effect of $\varphi$ on a polynomial $f \in R[X]$ is to change it to a Laurent polynomial in $T$ whose leading and lowest coefficient $=L(f)$ up to a sign. If $\varphi(\mathbf{v})=\left(\varphi\left(v_{1}\right), \ldots, \varphi\left(v_{n}\right)\right)$ and $m_{i}$ are least integers so chosen that $T^{m_{i}} \varphi\left(v_{i}\right)=w_{i} \in R[X, T]$, then $\mathbf{w}=\left(w_{1}, \ldots, w_{n}\right) \in \operatorname{Um}_{n}(R[X, T])$. Applying (2.1) and (2.3),

$$
\mathbf{w} \equiv \mathbf{w}(0) \quad\left(\bmod E_{n}(R[X, T])\right) \quad \text { and } \quad \mathbf{w}(0) \equiv L(\mathbf{v}) \quad\left(\bmod E_{n}(R)\right) .
$$

But then $\varphi(\mathbf{v}) \equiv L(\mathbf{v})\left(\bmod E_{n}(R[X, T])\right)$. Hence $\mathbf{v} \equiv L(\mathbf{v})\left(\bmod E_{n}(R[X])\right)$. 


\section{REFERENCES}

[B] H. Bass, Liberation des modules projectifs sur certains anneaux de polynomes (Sem. Bourbaki, 26 année, 1973/74, exp. 436-452), Lecture Notes in Math., vol. 431, Springer-Verlag, 1975,

[BCW] H. Bass. E. H. Connell and D. L. Wright, Locally polynomial algebras are symmetric algebras, Invent. Math. 38 (1977), 279-299.

[BR] S. M. Bhatwadekar and R. A. Rao, Efficient generation of ideals in polynomial extensions of an affine domain (preprint).

[L] T. Y. Lam, Serre's conjecture, Lecture Notes in Math., vol. 635, Springer-Verlag, 1978.

[M] S. Mandal, On efficient generation of ideals, Invent. Math. 75 (1984), 59-67.

[MK] N. Mohan Kumar, On two conjectures about polynomial rings, Invent. Math. 46 (1978), 225-236.

[S] A. A. Suslin, On the structure of the special linear-group over polynomial rings, Math. USSR-Izv. 11 (1977), 221-238.

School of Mathematics, Tata Institute of Fundamental Research, Colaba, Bombay 400005 , INDIA

Current address: Department of Mathematics, The University of Chicago, 5734 South University Avenue, Chicago, Illinois 60637 